\title{
The impact of choosing words carefully: an online investigation into imaging reporting strategies and best practice care for low back pain
}

\author{
Emma L Karran ${ }^{1,2}$, Yasmin Medalian ${ }^{1}$, Susan L Hillier ${ }^{1}$ ， G. Lorimer Moseley ${ }^{\text {Corresp. }{ }^{1,3}}$ \\ ${ }^{1}$ School of Health Sciences, University of South Australia, Adelaide, SA, Australia \\ 2 Royal Adelaide Hospital, Adelaide, South Australia, Australia \\ 3 Neuroscience Research Australia, Sydney, New South Wales, Australia \\ Corresponding Author: G. Lorimer Moseley \\ Email address: lorimer.moseley@unisa.edu.au
}

Background. Low back pain clinical practice guidelines consistently recommend against the routine ordering of spinal imaging - however imaging is frequently requested in primary care, without evidence of benefit. Imaging reports frequently identify degenerative features which are likely to be interpreted as 'abnormal' - despite their high prevalence in symptom-free individuals. The aim of this study was to investigate whether post-imaging back-related perceptions are influenced by providing prior information about normal findings, and to compare the effect of receiving imaging results with best practice care (without imaging). The impact of introducing novel, 'enhanced' reporting strategies was also explored.

Methods. This study was a simulated-patient, randomised, multiple-arm experiment. Patient scenarios were presented to volunteer healthy adult participants via an online survey. In the scenarios, 'virtual' patients with low back pain were randomised to one of three groups. Group 1 received imaging and was pre-informed about normal findings. Group 2 received imaging (without pre-information). Group 3 received best practice care quality information without imaging. Group 1 was further divided to receive either a standard report, or an 'enhanced' report (containing altered terminology and epidemiological information). The primary outcome was back-related perceptions (BRP) - a composite score derived from three numeric rating scale scores exploring perceptions of spinal condition, recovery concerns and planned activity. The secondary outcomes were satisfaction and kinesiophobia.

Results. Full data were available from 660 participants (68\% female). Analysis of covariance revealed a significant effect of group after controlling for baseline BRP scores $\left(F(2,74)=10.4, p<0.001, \eta_{p}^{2}=.04\right)$. Pairwise comparisons indicated that receiving best practice care resulted in more positive BRPs than receiving imaging results, and receiving 
prior information about normal findings had no impact. Enhanced reporting strategies also positively impacted BRPs $\left(F(1,275)=13.06, p<0.001, \eta_{p}{ }^{2}=.05\right)$. Significant relationships between group allocation and both satisfaction $\left(F(2,553)=7.5, p=0.001, \eta_{p}^{2}=.03\right)$ and kinaesiophobia $\left(F(2,553)=3.0, p=0.050, \eta_{p}{ }^{2}=.01\right)$ were found, with statistically significant pairwise comparisions again in favour of best-practice care.

Conclusion. Intervention strategies such as enhanced reporting methods and the provision of quality information (without imaging) have the potential to improve the outcome of patients with recent-onset LBP and should be further considered by primary care providers. 
1 The impact of choosing words carefully: an online investigation into imaging

2 reporting strategies and best practice care for low back pain

3 Emma L. Karran ${ }^{1,2}$, Yasmin Medalian ${ }^{1}$, Susan L. Hillier ${ }^{1}$, G. Lorimer Moseley ${ }^{1,3}$

4

$5{ }^{1}$ Sansom Institute for Health Research, University of South Australia, Adelaide, SA, Australia,

$6 \quad{ }^{2}$ Royal Adelaide Hospital, Adelaide, SA, Australia, ${ }^{3}$ Neuroscience Research Australia, Sydney,

7 NSW, Australia

\section{8}

9

10

Corresponding author:

G. Lorimer Moseley

11

12 University of South Australia GPO Box 2471, Adelaide, South Australia, 5001, Australia e: lorimer.moseley@unisa.edu.au 


\section{ABSTRACT}

Background. Low back pain clinical practice guidelines consistently recommend against the routine ordering of spinal imaging - however imaging is frequently requested in primary care, without evidence of benefit. Imaging reports frequently identify degenerative features which are likely to be interpreted as 'abnormal' - despite their high prevalence in symptom-free individuals. The aim of this study was to investigate whether post-imaging back-related perceptions are influenced by providing prior information about normal findings, and to compare the effect of receiving imaging results with the effect of receiving best practice care that does not include imaging. The impact of introducing novel, 'enhanced' reporting strategies was also explored.

Method. This study was a simulated-patient, randomised, multiple-arm experiment. Patient scenarios were presented to volunteer healthy adult participants via an online survey. In the scenarios, 'virtual' patients with low back pain were randomised to one of three groups. Group 1 received imaging and was pre-informed about normal findings. Group 2 received imaging (without pre-information). Group 3 received best practice care - quality information without imaging. Group 1 was further divided to receive either a standard report, or an 'enhanced' report (containing altered terminology and epidemiological information). The primary outcome was back-related perceptions (BRP) - a composite score derived from three numeric rating scale scores exploring perceptions of spinal condition, recovery concerns and planned activity. The secondary outcomes were satisfaction and kinesiophobia.

Results. Full data were available from 660 participants (68\% female). Analysis of covariance revealed a significant effect of group after controlling for baseline BRP scores $(F(2,74)=10.4$, $\left.\mathrm{p}<0.001, \mathrm{n}_{\mathrm{p}}{ }^{2}=.04\right)$. Pairwise comparisons indicated that receiving best practice care resulted in more positive BRPs than receiving imaging results, and receiving prior information about normal findings had no impact. Enhanced reporting strategies also positively impacted BRPs $\left(F(1,275)=13.06, p<0.001, \eta_{p}{ }^{2}=.05\right)$. Significant relationships between group allocation and both 
47 satisfaction $\left(F(2,553)=7.5, p=0.001, \eta_{p}{ }^{2}=.03\right)$ and kinaesiophobia $(F(2,553)=3.0, p=0.050, \eta$

$48 p^{2}=.01$ ) were found, with statistically significant pairwise comparisions again in favour of best49 practice care.

50

51 Conclusion. Intervention strategies such as enhanced reporting methods and the provision of quality information (without imaging) have the potential to improve the outcome of patients with recent-onset LBP and should be further considered by primary care providers.

\section{INTRODUCTION}

57

Low back pain (LBP) is a highly prevalent condition which is most often self-limiting and does not require investigations.(Maher et al. 2016) Current clinical practice guidelines for the management of recent-onset LBP outline specific circumstances in which spinal imaging should be considered(Chou et al. 2011; Koes et al. 2010) - but otherwise recommend against routine imaging. Despite this guideline consensus, spinal imaging is frequently requested by primary care providers(Dagenais et al. 2014; Williams et al. 2010) - most often inappropriately(Emery et al. 2013) and without evidence of benefit.(Chou et al. 2009)

General Practitioners (GP)s order spinal imaging for reasons including insufficient time to discuss the risks and benefits of scans, concerns regarding their vulnerability for malpractice and a desire to meet patient expectations.(Sears et al. 2016) Imaging is also often requested for the purpose of providing patients with reassurance.(Howard \& Wessely 1996) In principle, providing reassurance to patients presenting to primary care with recent-onset low back pain is a consistent recommendation in care guidelines.(Koes et al. 2010) However, while there is evidence for the reassuring value of medical investigations for some conditions,(Devcich et al. 2012; Howard et al. 2005) the reassuring potential of imaging for patients with LBP has not been demonstrated.(Rolfe \& Burton 2013) 
76 The failure of spinal imaging to reduce patient concern is hardly surprising when the high

77 prevalence of degenerative changes observed on the scans of asymptomatic adults is considered.(Brinjikji et al. 2014) Descriptions of these 'normal' changes in imaging reports (using terms suggestive of structural deterioration) are likely to have unwarranted and unnecessary effects on patient perceptions and behaviour.(Sloan \& Walsh 2010)

In 1998, Roland and van Tulder(Roland \& van Tulder 1998) suggested that: "Radiologists must take some responsibility for the way their reports are used and interpreted" (p. 230) in order to reduce the potential for harmful misinterpretation of imaging findings. A recent study (McCullough et al. 2012) has retrospectively examined the effect of including epidemiological information (i.e. a statement reporting the prevalence rates of common imaging findings in asymptomatic adults) alongside lumbar MRI reports - with some promising indications. Also of interest is evidence that providing patients with information about normal cardiac test results prior to their receipt of their own results - may optimise the reassuring potential of normal test findings.(Petrie et al. 2007)

In this online study, we investigated varied approaches to imaging reporting and their influence on patient perceptions regarding the condition of their back, concerns about recovery and plans to engage in activity. We also compared the reassuring value of receiving spinal imaging with the delivery of quality information without imaging, consistent with guideline-based 'best practice' care.

The primary aims of this study were:

i. To investigate whether post-imaging back-related perceptions are influenced by the provision of prior information about normal findings.

ii. To compare the effect of receiving imaging results with the effect of receiving best practice care that does not include imaging.

The secondary aims of this study were: 
104 i. To investigate the effect of receiving imaging results compared to best practice care (that 105 does not include imaging) on kinesiophobia.

106 ii. To investigate whether patients with back pain who receive best practice care from their GP are as satisfied as those who receive spinal imaging.

108 iii. To compare back-related perceptions following standard reporting of imaging findings with back-related perceptions following 'enhanced' reporting.

\section{METHODS}

114 This study was approved by the University of South Australia Human Research Ethics Committee (ID 0000035363). A study protocol was registered on Open Science Framework prior to completion of data collection (https://osf.io/4axy6/). Any deviations from that protocol are noted in this manuscript. The reporting of this study is consistent (wherever possible) with the CONSORT 2010 checklist (http://www.consort-statement.org/).

\section{Design and overview}

We used a simulated patient, 3-armed, randomised online experiment to investigate the impact of varied imaging reporting strategies and GP-delivered best practice care on patient cognitions, planned behaviour and satisfaction with healthcare. Adult volunteers were presented with an age-matched hypothetical patient scenario and requested to respond to survey questions as if they were the patient described. Participants were randomly allocated to the three study groups (via the survey software randomisation feature) and blinded to conditions other than their own.

\section{Participants}

We recruited participants via an online strategy involving email (using personal and professional networks of the authors), social media and website advertising. Participants were invited to "opt-in" to the study by following a link to the study information and confirmed 
133 consent through voluntarily commencing the survey. Individuals were eligible for inclusion if

134 they were aged over 18 years and had sufficient English language proficiency to complete the 135 questionnaire.

136

\section{Procedure}

138 Participants used their personal computers or smartphones to access the survey via web-based 139 survey software. They completed a 4-item demographic questionnaire and 6 baseline screening questions, and were provided with a scenario describing a person who injures their back while lifting. Participants were asked to imagine that they were person described in the scenario as they completed the baseline primary outcome measures. The scenario was developed to describe the person's concern about their slow recovery ( 3 weeks later) and their decision to go to see their GP. Participants were randomly allocated to 3 groups:

i. A scan is recommended and the GP provides information about normal scan findings

ii. A scan is recommended (but no information about normal findings is provided)

iii. The GP provides best practice care (and does not recommend a scan).

Patients who received a scan were further randomised to a type of reporting strategy as detailed in Figure 1. All participants then completed the primary and secondary outcome measures. Details of the manipulations (and examples) are provided in Supplement 1.

\section{Measures}

\section{Outcome measures}

154 The primary outcome measure was a composite score derived from 3 numeric rating scale (NRS) responses (see Supplement 2). The summed outcome score was labelled the BackRelated Perceptions (BRP) score, with higher scores indicating more positive perceptions. We considered that 'patient' reassurance (reduced fear and concern) would be reflected by higher BRP scores. Reassurance is variably defined and measured; we considered that a change in reassurance would be more likely to be detected by a composite outcome measure than a single NRS question. 
162 The two secondary outcome measures were the Tampa Scale of Kinesphobia (TSK-11)(Tkachuk

163 \& Harris 2012) and a patient satisfaction rating (see Supplement 3).

164

165

166

167

168

169

170

171

172

173

174

175

176

177

178

179

180

181

182

183

184

185

186

187

188

189

\section{Demographics and baseline screening}

Self-report data were collected on age, gender, country of residence, language, and education level attained. Participants completed the Back Pain Attitudes Questionnaire(Darlow et al. 2014) (at baseline) and were asked to indicate "yes" or "no" responses to questions relating to previous back pain, recent back pain, current 'chronic' back pain and history of back scans.

\section{Data Analysis}

Sample size calculation

The study was powered to detect a small effect size,(Cohen 1998) with a power of $80 \%$ and an alpha value of 0.05 . Assuming a correlation 0.7 , the minimum sample size required for each of the 3 groups was 195 participants.

\section{Statistical analysis plan}

To address each of the primary research aims an analysis of covariance (ANCOVA) was conducted to determine if there was a significant effect of group on the primary outcome (BRP scores) after controlling for the effect of baseline BRP scores. Tukey corrected post-hoc pairwise comparisons (with bootstrapped significance and confidence intervals) investigated whether there was a significant difference between the best practice group and either (or both) of the imaging groups, and between the two imaging groups.

To address the secondary research aims (i) and (ii) we performed one-way analysis of variance (ANOVA) to determine the relationship between the group variable and the secondary outcome measures. If a significant effect was identified, Tukey's post-hoc tests with Bonferroni correction were conducted to identify the between-group differences. 
190

191

192

193

194

195

196

197

198

199

200

201

202

203

204

205

206

207

208

209

210

211

212

213

214

215

216

217

To address secondary research aim (iii) we used ANCOVA to investigate whether there was a significant effect of group after controlling for the effect of baseline BRP scores. The level of significance for this sub-study was set at $\mathrm{P} \leq 0.025$ to adjust for multiple comparisons. The level of significance for all other studies was set at $P \leq 0.05$. Statistical analyses was undertaken using SPSS Statistics software (v22.0.0.0, IBM Corporation, New York).

\section{RESULTS}

\section{Sample characteristics}

788 participants commenced the online study between February and June, 2017. 660 participants completed baseline and post-intervention BRP scores and were included in the analysis. The baseline characteristics of included participants (including mean scores for the Back Pain Attitudes Questionnaire) are presented in Table 1.

\section{Experimental effects}

Table 2 presents mean scores on the primary and secondary outcome measures across experimental groups, and change scores on the primary outcome.

'Pre-information' (+ scan) vs 'no pre-information' (+ scan) vs 'best practice'

ANCOVA was conducted to examine the effect of group on BRP whilst controlling for baseline BRP scores. Levene's test and normality checks were carried out and assumptions were met. After adjusting for baseline BRP scores there was a significant difference between the 3 groups in post intervention BRP scores $\left(F(2,74)=10.4, p<0.001, \eta_{p}{ }^{2}=.04\right)$. Post-hoc pairwise comparisons revealed significant differences between the 'no pre-information' group and the 'best practice' group ( $p=0.001, d=.33)$, and between the 'pre-information' group and the 'best practice' group ( $p=0.002, d=.47$ ). There was no significant difference in BRP between the 2 groups who received scans $(p=0.202)$. For both of the significant comparisons, mean BRP 
218 scores (indicating more positive perceptions) were higher for the group who received best 219 practice care.

220

221 One-way ANOVA revealed a significant relationship between group and kinesiophobia

$222\left(\mathrm{~F}(2,553)=3.0, \mathrm{p}=0.050, \eta_{\mathrm{p}}{ }^{2}=.01\right)$. Post-hoc pairwise comparisons revealed a significant

223 difference between the 'no pre-information' group and the 'best practice' group ( $p=0.044$,

$224 \mathrm{~d}=.29)$. Mean kinesiophobia scores were lower for the group who received best-practice care.

225

226 There was a significant relationship between group and satisfaction ratings $(F(2,553)=7.5$,

$\left.227 \mathrm{p}=0.001, \eta_{\mathrm{p}}{ }^{2}=.03\right)$. Post-hoc pairwise comparisons revealed significant differences between the

228 'no pre-information' group and the 'best practice' group ( $p=0.009, d=.34)$, and between the

229 'pre-information' group and the 'best practice' group $(p=0.001, d=.33)$. Mean satisfaction

230 scores were higher for those who received best practice care.

231

232 Standard reporting vs enhanced reporting

233 There was a significant effect of group on BRP after controlling for the effect of baseline BRP

234 scores $\left(F(1,275)=13.06, p<0.001, \eta_{p}{ }^{2}=.05\right)$. Mean BRP scores were higher for those who

235 received enhanced reporting.

236

237 The individual effects of the two components of enhanced reporting were also examined.

238 When compared with standard reporting, the effect of altering the summary terminology

239 (alone) was significant $\left(F(1,221)=7.70, p=0.006, \eta_{p}^{2}=.03\right)$ as was the effect of both altering the

240 summary terminology and including epidemiological information $(F(1,207)=8.40, p=0.004, \eta$

$\left.241 \mathrm{p}^{2}=.04\right)$. There was no statistically significant difference between the two versions of enhanced

242 reporting practices $(p=0.85)$.

243

244

245

DISCUSSION

246 
247 Summary

248 This investigation examined the impact of novel spinal imaging reporting practices on 'virtual'

249 patient BRPs, and provided comparison with best-practice care that does not include imaging.

250 In summary, receiving high quality information (without imaging) resulted in more positive

251 BRPs than receiving imaging results, and was associated with higher satisfaction. Small-to-

252 medium effect sizes indicate the importance of these findings. For patients who were referred

253 for spinal imaging - altering the terminology of the report summary and including

254 epidemiological information on the report improved perceptions, but whether or not

255 information about normal findings was delivered prior to receiving an imaging report had no

256 impact.

257

258 Strengths and limitations

259 This online study was a novel approach which accessed a large adult population. To our

260 knowledge it is the first investigation to directly compare the reassuring potential of spinal

261 imaging with best practice care, and consider the impact of varied reporting strategies in a

262 randomised design. There are however, some weaknesses with the online scenario-based

263 study design. Participant engagement in the task and understanding of the questions could not

264 be monitored. The usefulness of participants imagining that they were the person described in

265 the scenario is uncertain and issues relating to the reliability and validity of this approach are not known. Participants may have had difficulty relating to the patient described, but that $94 \%$

267 reported previous back pain might suggest that they were able to partially identify with the

268 'patient' experience. Not collecting this information and more detailed data on participant

269 characteristics were shortcomings of this study.

270

271 There may also be limitations related to using the primary outcome measure (BRP) as a proxy

272 for reassurance, and the identification of patients' planned behaviour (which does not offer

273 information about how someone would actually behave in a real situation). In addition - BRP

274 was an outcome measure developed specifically for this study and has not undergone 
275 psychometric evaluation. This limitation should be considered when interpreting the strength

276 of this study's findings.

277

278 The potential for recruitment bias associated with using the authors' networks to recruit

279 participants was minimised by instructing individuals who were likely to be knowledgeable

280 about 'pain' or 'imaging' to not complete the study themselves - but to disseminate the

281 advertisement to uninformed members of their own networks. However, the high proportion

282 of University educated participants (80\%) suggests that the study population is not

283 representative of the general population. The supposed higher health literacy and cognitive

284 ability of study participants is likely to have impacted their interpretation of information and

285 may limit study generalisability.

286

287

Relevance to existing literature

288

Previous studies have suggested that diagnostic tests for some conditions can offer reassurance, but evidence for the reassuring value of imaging for LBP has been lacking. This study cautiously supports that spinal imaging results - at least when reported in a 'standard' fashion - are not in themselves reassuring. Findings are also consistent with recent indications that adopting 'enhanced' reporting practices (such as including epidemiological information) can offer benefit(McCullough et al. 2012) and supports the view of Roland and van Tulder (1998)(Roland \& van Tulder 1998) that attention to the content and language of spinal imaging reports is warranted. A current randomised trial investigating the inclusion of epidemiological information in lumbar spine imaging reports is likely to further inform the need for practice change.(Jarvik et al. 2015)

Implications

The findings of this study have the potential to beneficially inform future health care delivery. General Practitioners are likely to be able to reassure patients with recent-onset LBP more effectively if they offer carefully considered information than if they order a scan. This finding 303 challenges the frequent belief that spinal imaging (for patients with a low likelihood of serious 
304 pathology) offers opportunity to alleviate patients' fears and concerns.(Howard \& Wessely 305 1996; Rolfe \& Burton 2013) Further to this, the suggestion that patients are also likely to be

306

307

308

309

310

311

312

313

314

315

316

317

318

319

320

321

322

323

324

325

326

327

328

329

330

331

332

more satisfied with this approach offers that avoidance of a scan is likely to be readily accepted by patients if high-quality information is provided. It is worthwhile acknowledging that this study did not investigate the effect of offering both scans and information. This approach which is probably common in clinical practice - should be addressed in future studies.

Kinaesiophobia was found to be lower for the 'best practice' group than for the 'no preinformation' group - however a four-point decrease in TSK-11 scores has been suggested to be required to reflect a clinically important reduction in fear of movement.(Woby et al. 2005) The two-point between-group difference observed in this study, while statistically significant, may not represent a meaningful difference. Further, although our randomised design and a priori sample size justified not collecting baseline TSK scores, and the BPAQ data showed no difference between groups on that measure at baseline, the between-group differences for TSK and for patient satisfaction were small and we cannot completely eliminate the possibility that they reflect baseline differences. As such, they should be interpreted with caution.

This exploratory study suggests that simply offering best practice care is indeed likely to be effective management for recent-onset LBP. That LBP outcomes are not, in general, improving may indicate limited application of the current recommendations or a need to offer clinicians further guidance regarding the provision of quality information. We consider that the information provided to participants in this study (see Supplement 1) (guided by current literature (TOP 2015; NICE 2016; Moseley \& Butler 2015; Stochkendahl et al. 2017; Traeger et al. 2017)) may not represent routine management and that the potential to improve best practice care currently exists.

This study also suggests that dedicating further attention to the content of spinal imaging reports is likely to be worthwhile. Providing explanations of 'normal' age-related features, carefully adapting summary terminology and accurately interpreting imaging findings offer the 
333 potential to positively impact patients' beliefs and concerns, and their subsequent engagement 334 in activity.

335

336

337

338

339

340

341

342

343

344

345

346

347

348

349

350

351

352

353

354

355

356

357

358

359

360

361

\section{REFERENCES}

Brinjikji W, Luetmer P, Comstock B, Bresnahan B, Chen L, Deyo R, Halabi S, Turner J, Avins A, and James K. 2014. Systematic Literature Review of Imaging Features of Spinal Degeneration in Asymptomatic Populations. American Journal of Neuroradiology.

Chou R, Fu R, Carrino JA, and Deyo RA. 2009. Imaging strategies for low-back pain: systematic review and meta-analysis. The lancet 373:463-472.

Chou R, Qaseem A, Owens DK, and Shekelle P. 2011. Diagnostic imaging for low back pain: advice for high-value health care from the American College of Physicians. Annals of internal medicine 154:181-189.

Cohen J. 1998. Statistical power analysis for the behavioral sciences. New Jersey: Lawrence Erlbaum.

Dagenais S, Galloway EK, and Roffey DM. 2014. A systematic review of diagnostic imaging use for low back pain in the United States. The Spine Journal 14:1036-1048.

Darlow B, Perry M, Mathieson F, Stanley J, Melloh M, Marsh R, Baxter GD, and Dowell A. 2014. The development and exploratory analysis of the Back Pain Attitudes Questionnaire (Back-PAQ). BMJ open 4:e005251.

Devcich DA, Ellis CJ, Broadbent E, Gamble G, and Petrie KJ. 2012. The psychological impact of test results following diagnostic coronary CT angiography. Health Psychology 31:738.

Emery DJ, Shojania KG, Forster AJ, Mojaverian N, and Feasby TE. 2013. Overuse of magnetic resonance imaging. JAMA internal medicine 173:823-825.

Howard L, Wessely S, Leese M, Page L, McCrone P, Husain K, Tong J, and Dowson A. 2005. Are investigations anxiolytic or anxiogenic? A randomised controlled trial of neuroimaging to provide reassurance in chronic daily headache. Journal of Neurology, Neurosurgery \& Psychiatry 76:1558-1564. 
362 Howard LM, and Wessely S. 1996. Reappraising reassurance-the role of investigations. Journal 363 of psychosomatic research 41:307-311.

364 Jarvik JG, Comstock BA, James KT, Avins AL, Bresnahan BW, Deyo RA, Luetmer PH, Friedly JL, 365 Meier EN, and Cherkin DC. 2015. Lumbar Imaging with Reporting of Epidemiology

366

367

368

369

370

371

372

373

374

375

376

377

378

379

380

381

382

383

384

385

386

387

388 (LIRE)-protocol for a pragmatic cluster randomized trial. Contemporary clinical trials 45:157-163.

Koes BW, van Tulder M, Lin C-WC, Macedo LG, McAuley J, and Maher C. 2010. An updated overview of clinical guidelines for the management of non-specific low back pain in primary care. Eur Spine J 19:2075-2094.

Maher C, Underwood M, and Buchbinder R. 2016. Non-specific low back pain. The Lancet. 10.1016/S0140-6736(16)30970-9.

McCullough BJ, Johnson GR, Martin BI, and Jarvik JG. 2012. Lumbar MR imaging and reporting epidemiology: do epidemiologic data in reports affect clinical management? Radiology 262:941-946.

Moseley GL, and Butler DS. 2015. Fifteen years of explaining pain: the past, present, and future. The Journal of Pain 16:807-813.

(NICE) National Guideline Centre (UK). 2016. Low back pain and sciatica in over 16s: assessment and management. Manchester: National Institute for Health and Care Excellence. https://www.nice.org.uk/guidance/ng59

Petrie KJ, Muller JT, Schirmbeck F, Donkin L, Broadbent E, Ellis CJ, Gamble G, and Rief W. 2007. Effect of providing information about normal test results on patients' reassurance: randomised controlled trial. BMJ 334:352.

Roland M, and van Tulder M. 1998. Should radiologists change the way they report plain radiography of the spine? The lancet 352:229-230.

Rolfe A, and Burton C. 2013. Reassurance after diagnostic testing with a low pretest probability of serious disease: systematic review and meta-analysis. JAMA internal medicine 173:407-416. 
389

390

391

392

393

394

395

396

397

398

399

400

401

402

403

404

405

406

407

408

409

410

411

412

Sears ED, Caverly TJ, Kullgren JT, and et al. 2016. Clinicians' perceptions of barriers to avoiding inappropriate imaging for low back pain-knowing is not enough. JAMA Internal Medicine. 10.1001/jamainternmed.2016.6364

Sloan TJ, and Walsh DA. 2010. Explanatory and diagnostic labels and perceived prognosis in chronic low back pain. Spine 35:E1120-E1125.

Stochkendahl MJ, Kjaer P, Hartvigsen J, Kongsted A, Aaboe J, Andersen M, Andersen M $\varnothing$, Fournier G, Højgaard B, and Jensen MB. 2017. National Clinical Guidelines for nonsurgical treatment of patients with recent onset low back pain or lumbar radiculopathy. European Spine Journal:1-16.

SurveyMonkey Inc. San Mateo, California, USA. Available from:

\section{https://www.surveymonkey.com/}

Tkachuk GA, and Harris CA. 2012. Psychometric Properties of the Tampa Scale for Kinesiophobia-11 (TSK-11). The Journal of Pain 13:970-977. http://dx.doi.org/10.1016/j.jpain.2012.07.001

(TOP) Toward Optimized Practice, Low Back Pain Working Group. 2015. Evidence-informed primary care management of low back pain: clinical practice guideline. Edmonton, $A B$. Available from: http://www.topalbertadoctors.org/cpgs/885801.

Traeger AC, O'Hagan ET, Cashin A, and McAuley JH. 2017. Reassurance for patients with nonspecific conditions-a user's guide. Brazilian Journal of Physical Therapy 21:1-6.

Williams CM, Maher CG, Hancock MJ, McAuley JH, McLachlan AJ, Britt H, Fahridin S, Harrison C, and Latimer J. 2010. Low back pain and best practice care: A survey of general practice physicians. Archives of internal medicine 170:271-277.

Woby SR, Roach NK, Urmston M, and Watson PJ. 2005. Psychometric properties of the TSK-11: a shortened version of the Tampa Scale for Kinesiophobia. Pain 117:137-144. 
Table $\mathbf{1}$ (on next page)

Participant characteristics 


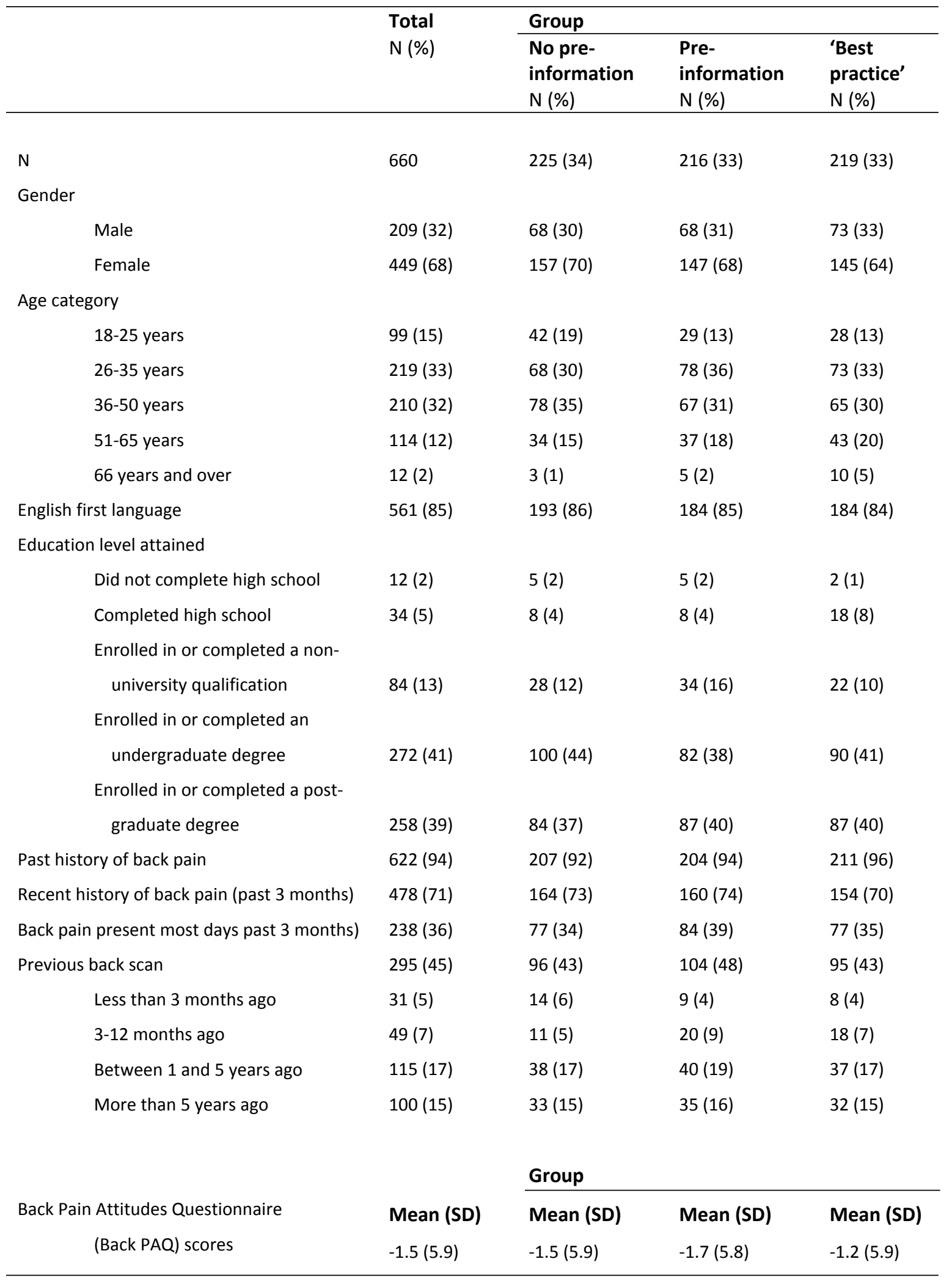

2 
Table 2 (on next page)

Primary and secondary outcome scores 
1 Table 2: Primary and secondary outcome scores

\begin{tabular}{|c|c|c|c|c|c|c|c|}
\hline \multicolumn{2}{|c|}{ Group } & $\mathbf{N}$ & $\begin{array}{r}\text { Baseline BRP } \\
\text { Mean (SD) }\end{array}$ & $\begin{array}{r}\text { Post-intervention } \\
\text { BRP } \\
\text { Mean (SD) }\end{array}$ & $\begin{array}{r}\text { BRP Change } \\
\text { score }\end{array}$ & TSK-11 & $\begin{array}{r}\text { Patien } \\
\text { satisfactior } \\
\text { rating }\end{array}$ \\
\hline 1. & No pre-information (+scan) & 225 & $18.9(5.5)$ & $20.7(5.8)$ & $1.8(4.4)$ & - & - \\
\hline & $\begin{array}{l}\text { Standard report } \pm \text { epidemiological } \\
\text { information }\end{array}$ & 121 & $18.7(5.6)$ & $19.8(6.0)$ & $1.1(4.4)$ & $24.0(7.0)$ & $6.2(2.4)$ \\
\hline 2. & $\begin{array}{l}\text { Pre-information (+scan) } \\
\text { (Standard report } \pm \\
\text { epidemiological information) }\end{array}$ & 216 & $18.5(5.5)$ & $20.3(5.8)$ & $1.8(5.0)$ & $23.0(7.8)$ & $6.2(2.3)$ \\
\hline 3. & 'Best practice' (no scan) & 219 & $19.2(6.0)$ & $22.2(5.4)$ & $3.0(4.8)$ & $22.0(7.1)$ & $7.0(2.4)$ \\
\hline 4. & Standard report & 165 & $18.4(5.6)$ & $19.2(6.0)$ & $0.9(4.7)$ & - & - \\
\hline 5. & $\begin{array}{l}\text { Standard report + epidemiological } \\
\text { information }\end{array}$ & 174 & $18.9(5.5)$ & $21.0(5.7)$ & $2.1(4.7)$ & - & - \\
\hline 6. & Altered summary terminology & 59 & $19.9(5.6)$ & $22.2(5.7)$ & $2.3(4.4)$ & - & - \\
\hline 7. & $\begin{array}{l}\text { Altered summary terminology + } \\
\text { epidemiological information }\end{array}$ & 45 & $18.4(4.8)$ & $21.4(4.7)$ & $3.0(4.8)$ & - & - \\
\hline 8. & $\begin{array}{l}\text { Enhanced report (altered } \\
\text { summary terminology }+/- \\
\text { epidemiological information) }\end{array}$ & 104 & $19.3(5.3)$ & $21.8(6.0)$ & $2.6(4.4)$ & - & - \\
\hline
\end{tabular}

2 


\section{Figure 1}

\section{Online study set-up}

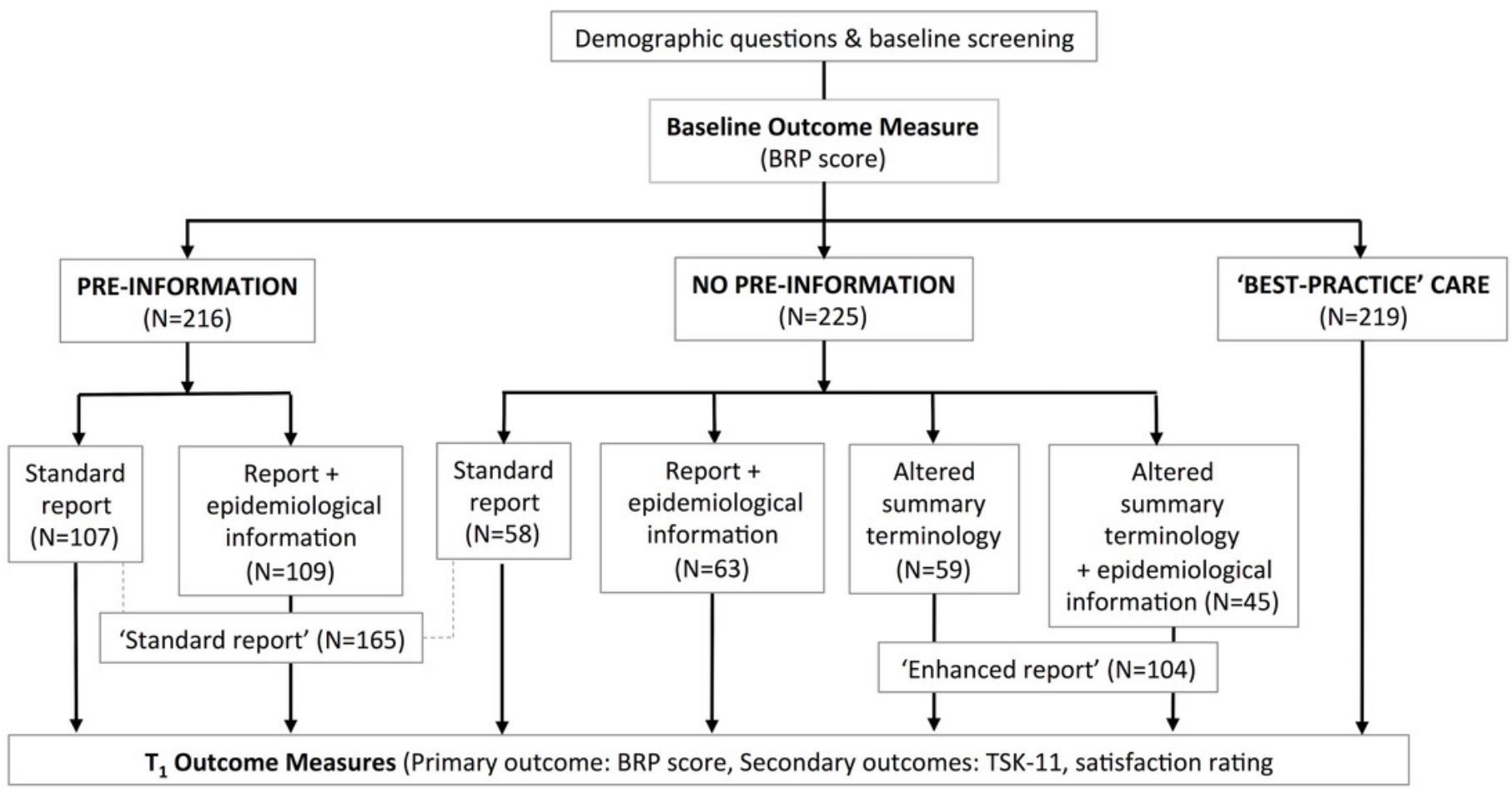

